Proceedings of the 2010 Winter Simulation Conference

B. Johansson, S. Jain, J. Montoya-Torres, J. Hugan, and E. Yücesan, eds.

\title{
MACHINE CONTROL LEVEL SIMULATION OF AN AS/RS IN THE AUTOMOTIVE INDUSTRY
}

\author{
Min S. Ko \\ Hye S. Shin \\ Dept. of Industrial Engineering \\ Paldalgwan-822, Ajou University \\ Suwon, 443-749, SOUTH KOREA
}

\author{
G.N. Wang \\ Sang C. Park \\ Dept. of Industrial Engineering \\ Paldalgwan-822, Ajou University \\ Suwon, 443-749, SOUTH KOREA
}

\begin{abstract}
This paper illustrates a case study of a PLC (Programmable Logical Controller) logic simulation in the car manufacturing industry. The case study is developed in order to simulate and verify the PLC control program for an automobile panel AS/RS. Because of increasing demand, the complexity of the supply system is rising in this industry. To cope with this problem, companies use AS (automated storage) and RS (retrieval system) systems, despite inherent logical complexities. Industrial automated processes use PLC code to control the AS/RS; however, control information and the control code (PLC code) are difficult to understand. Therefore, this paper suggests a PLC simulation environment, using 3D models and PLC code, which consists of real automobile manufacturing data. Data used in this simulation is based on 3D and logical models, using actual size and PLC signals, respectively. The environment resembles a real factory; users can verify and test the PLC code using the simulation prior to implementation of AS/RS. The developed simulation environment can be used to run AS/RS systems to reduce time and cost.
\end{abstract}

\section{INTRODUCTION}

To fulfill increasing customer demand for various requirements, small quantity batch production and JIT (Just in Time) systems have been introduced in modern industries. The production system information and logistics have to comprise a single body to introduce these systems and thus high-speed facilities and high-accuracy control are necessary. The AS/RS (Automated Storage and Retrieval System), a rapid logistic system in automated manufacturing systems, plays a significant role to carry out production of parts and allow flexibility in functional changes.

An AS/RS is a combination of equipment and controls that handle, store, and retrieve materials as needed with precision, accuracy, and speed under a defined degree of automation. Systems vary from relatively simple, manually controlled order-picking machines operating in small storage structures to extremely large, computer-controlled storage/ retrieval systems totally integrated into a manufacturing and distribution process. The AS/RS is applied to increase production volume in automated manufacturing systems through automation of facility control and strengthening of stock control. Application of the technology of AS/RS is a kernel skill for the construction of automated logistics, FMS, and CIM. Systematical management of information is not easily realized in an AS/RS, because special control rules and communication code are utilized to control the AS/RS, which uses an exclusive controller (Han et al. 1987, Hausma et al. 1976). 


\section{Ko, Shin, Wang, and Park}

Various panels are used in different car types and many kinds of cars are typically produced in an automobile factory. Accordingly, rapid and accurate response is needed for the automated manufacturing system in the automotive industry. However, because of high complexity control logic makes it is challenging to make and validate the control code, as in the case of PLC (Programmable Logical Controller), which is written with a low level language and depends on the worker's experience. A great deal of time and effort are expended in validating the control code in the implementation and test-run process of an AS/RS (Lee and Roh 2006).

The objective of this paper is to propose a construction method of a PLC validation simulation environment for an AS/RS in the automobile industry. The proposed approach is different from the metamodel simulation (Kang et al. 2006) or a logical validation simulation using DES (Discrete Event Simulation) (Kim 1994, UDMTek 2008). In the proposed method, real data, not only a logical model but also 3D models actually used in the industry, is employed to bridge the gap between the simulation environment and the real field. This paper addressed the following three issues:

- A direct link between the simulation model and real data

- Realistic representation of the simulation model

- Increased utilization of the simulation model.

Direct link relation means that the simulation models can be used in the factory without any additional work while realistic representation means that 3D models, used in the simulation, are based on the real design parameters rather than simplified data. This paper presents an efficient construction methodology for a virtual factory to validate PLC code using a logical model and a virtual 3D model. PLC code used in AS/RS is validated under the virtual factory before the design and construction of an automobile factory based in the USA.

Chapter 2 describes the working process of the AS/RS and the applied methodology. Chapter 3 defines the logical model then chapter 4 describes how to make a 3D virtual factory for simulation using the logical model. Finally, concluding remarks are provided in chapter 5.

\section{THE WORKING PROCESS OF AS/RS}

The remarkable revival of the automobile industry generalized a production system of multi-type vehicle. The robots, fixtures, and AGVs plays different roles in a car code informed from PLC. This code makes it possible to coordinate jobs according to car type in a single line. The car assembly line is more complicated than other manufacturing lines, because various panels must be moved to correct positions with precise timing.

\subsection{Outline of AS/RS Simulation}

The purpose of the AS/RS simulation is to reduce logical errors and to validate the PLC code before the test run. Figure 1 is an outline of the simulation proposed in this paper.

(1) Analyze the process: The process flow and the PLC program have to be analyzed to understand the operation sequence. A logical model is used in this simulation and the total operation sequences are designed through this step.

(2) Construct the virtual factory: A virtual plant model is constructed. The model consists of many kinds of virtual devices that imitate real devices in the manufacturing line so that users can easily verify the PLC program. To make a virtual device, 3D models have to be corrected and corres- 
ponding kinematic information is inserted into the 3D models so that they will move like the real devices. A logical model receives an input signal from the PLC code controls the 3D model in the virtual plant.

(3) Construct the control system: To construct the control system, the PLC code that controls the devices has to be developed after analyzing the process. If the control system is similar to a previously developed control system, the code could be reused after modifying parts.

(4) Test the manual working mode: The PLC code of each device has to be tested using a logical model and a virtual plant model in this step (manual working mode). The user conducts a test of wherein the signal connected with the PLC is changed and then observes the variation of the output signal. A logical model is connected with motions contained in the virtual plant model. When an event occurs that translates the logical model state from the before-state to after-state, the corresponding motion is designed to occur at the same time.

(5) Test the auto working mode: The auto working mode means that all devices work automatically according to the PLC code. The PLC code is tested before an auto mode test run in an environment that consists of a HMI (Human Machine Interface), a hardware PLC, the logical model, and a virtual plant. The proposed method creates an environment corresponding with that of the PLC code validation process in the actual manufacturing line, because it uses actual PLC code and a plant model reflecting the actual size used in the real factory. As such, it is possible to validate logic and motion of the AS/RS before execution of the test run.

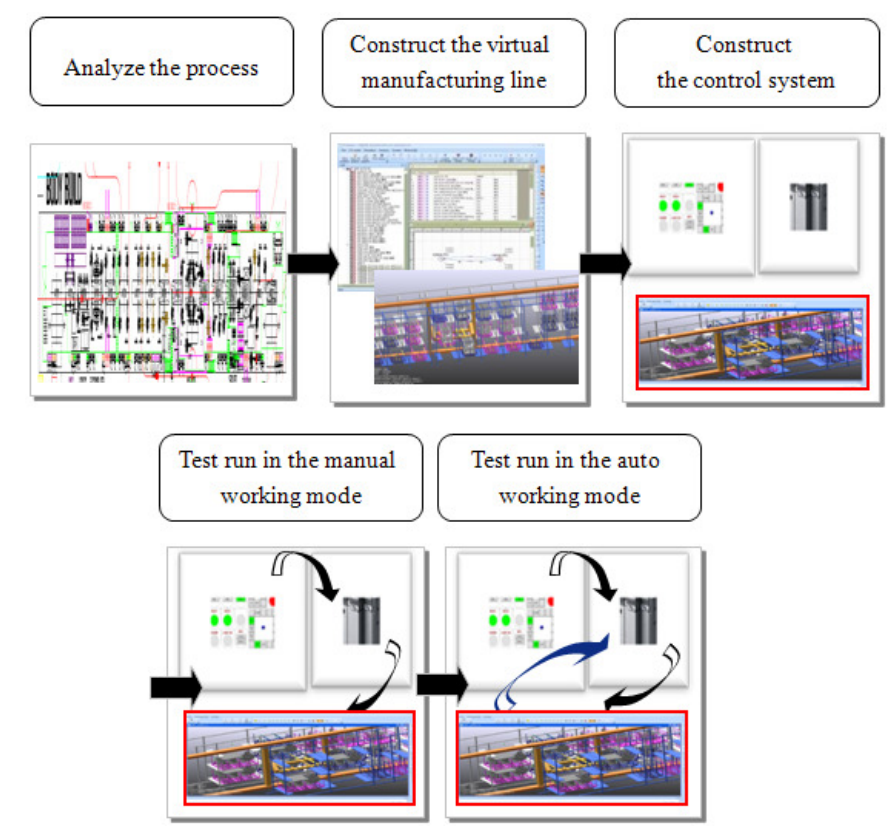

Figure 1: Procedure of constructing PLC code validation environment of AS/RS

\subsection{Construction of an AS/RS in an Automobile Assembly Line}

Generally, the AS/RS used in automobile manufacturing systems consist of the following 5 elements:

(1) Pallet: The pallet plays a role of a package for translation of the panel. It contains a certain number of panels, and its state is divided into "Full Pallet" and "Empty Pallet". If an "empty pallet 
occurs" signal is triggered by a sensor when an empty pallet is put in the Rack (IN), then the PLC sends a signal to the stoker-crane indicating that a pallet has to be replaced. A pallet filled with panels is classified according to shape, size, and color, because it contains unique kinds of panels identified by size, shape, and car type.

(2) Rack: The rack is a kind of multi-floor storage where the pallets are located for use in the AS/RS. Each rack room has its own address to identify the position in the rack, and the rack has two sides, Rack (IN) side and Rack (OUT). The forklift inserts a full pallet and pulls off an empty pallet through the Rack (IN) and the handling robot selects a panel from the pallet at the Rack (OUT) side.

(3) Forklift: A forklift replaces an empty pallet with a full pallet at the Rack (IN) side.

(4) Stoker-Crane: A stoker crane is kind of a transporter to carry a full pallet from Rack (IN) to Rack (OUT) or an empty pallet from Rack (OUT) to Rack (IN). It has 3 kinds of motors to move in the $\mathrm{x}, \mathrm{y}$ and $\mathrm{z}$ directions, and the PLC sends an integer signal to indicate the rack address.

(5) Handling Robot: The handling robot selects a panel from the pallet located in the Rack (OUT) side. When the handling robot selects the final panel from a pallet, it sends a signal to the PLC to inform the location of the empty pallet.

The proposed simulation uses these 5 elements to construct the AS/RS environment for validation of the PLC code.

Each pallet has a data structure as outlined in Table 1. Four kinds of tags indentify the pallet state and the position of a pallet. For example, $\{\mathrm{A} 1$, Back, Full, $(1,1,1)\}$ means that "A full pallet filled with car type A1's Back Panels" is located in RACK (OUT), 1st row 1st column.

Table 1: Data structure of a pallet

\begin{tabular}{|c|c|c|}
\hline TAG & DECLARATION & CONTENT \\
\hline Car type information & "A1", "A2",.., "A4" & The code of type of a car \\
\hline Panel type & "Back", "Front",..,"Cowl" & Information of a part of a car \\
\hline Panel existence & "Full", "Empty" & $\begin{array}{l}\text { Full: All panels are filled in a pallet } \\
\text { Empty: No panel is filled in a pallet }\end{array}$ \\
\hline Position address & $(\mathrm{X}, \mathrm{Y}, \mathrm{Z})$ & $\begin{array}{l}\text { X: Integer value of an address "X-axis" } \\
\text { Y: Integer value of an address "Y-axis" } \\
\text { Z: Rack(IN) }=0 \text {, Rack (OUT) }=1\end{array}$ \\
\hline
\end{tabular}

\subsection{Assumptions Employed in the AS/RS Simulation}

The AS/RS system comprises a huge facility in a car assembly line. This paper makes some assumptions regarding limitations of the simulation range and physical motion so as to increase the efficiency and reality of the simulation.

(1) The forklift motion is not considered in this simulation. The motions alternate with user defined events, because logic validation of the AS/RS is the main focus of this simulation.

(2) The panel selected by the handling robot is not considered in this simulation. To take this situation into consideration, it is substituted with an event in this simulation. That is, when the robot selects the last panel from a pallet, it sends a signal "(x, y, z) pallet is empty now" to the PLC. 
Under these two assumptions the range of the simulation is limited in the PLC code validation using a 3D model. Therefore, forklift motion and removal of panels by the handling robot are replaced with events.

\subsection{AS/RS Process}

Figure 2 illustrates the process of logic transaction and points of concern a pallet in the AS/RS.

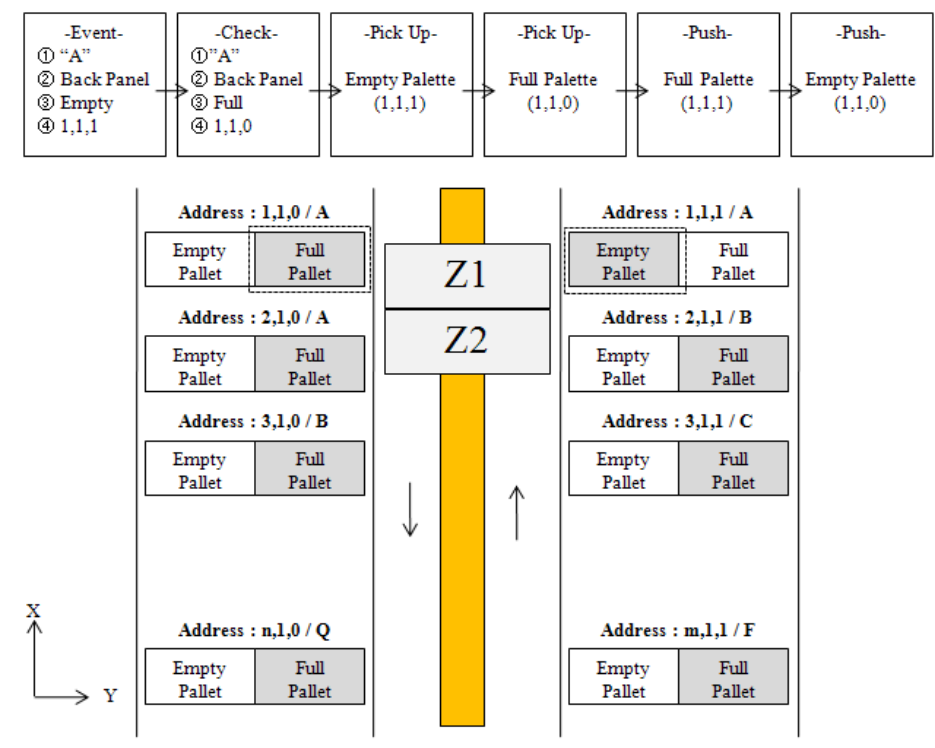

Figure 2: Process of the AS/RS

The points of concern are : (1) Car Type (2) Kinds of Panel (3) Rack Sides (4) Full or Empty state of a pallet, as shown in Table. 1. The logic transaction of a pallet has 6 steps. The stocker-crane is located in a "Stand by State" either before step 1 or after step 6. The following is an example of the proposed logic transaction process.

(1) Event: A robot selects the last back panel of car type "A" from the pallet located in address $(1,1$, 1), and then sends a signal indicating an empty pallet state to the PLC.

(2) Check: The PLC checks whether it is possible to obtain a full pallet of a back panel from the pallet address $(1,1,0)$. If a full pallet is located in address $(1,1,0)$, a position sensor sends a signal to the PLC. If a full pallet is not there, the PLC informs the state of the pallet to the worker through the HMI.

(3) Pick Up (Empty): The Stoker-Crane approaches the address $(1,1,1)$ where the empty pallet is located and then $\mathrm{Z1}$ reaches the pallet to load.

(4) Pick Up (Full): The Stoker-Crane approaches to the address $(1,1,0)$ where the full pallet located and then $\mathrm{Z} 2$ reaches the full pallet for loading.

(5) Push (Full): A full pallet located in $\mathrm{Z} 2$ is unloaded at the address $(1,1,1)$ and then a position signal is sent to the PLC. The handling robot goes to $(1,1,1)$ to select a new panel after receiving a signal from the PLC.

(6) Push (Empty): An empty pallet located in Z1 is unloaded at the address $(1,1,0)$ and then a forklift withdraws the pallet throughout from Rack (IN). 
These 6 steps show the procedure when the stoker-crane replaces an empty pallet located at the Rack (OUT) side with a full pallet located at the Rack (IN) side. A single pallet relocation simulation is not complex; however, if many pallets are moved at the same time, the logic complexity is increased. The reasons for this are as follows: first, it is not possible to observe the moving pallet between racks, because the AS/RS logic process is drawn in 2D. Second, it is difficult to simulate manual events (pallet creation, pallet deletion) on the PLC code.

This manual simulation consumes too much time in the manual test run step when a worker wishes to create an event to test the PLC. This paper proposes a simulation environment for validating the PLC code using logical models and 3D models to view the state change of a pallet and the motion of a stoker crane resulting from an event. The proposed method makes it easier to understand the process of AS/RS and reduces the time required to debug PLC code.

\subsection{Method of Constructing Simulation Environment}

The PLC plays a role similar to a traffic light in the manufacturing line. The traffic light of an intersection located in a busy region controls the time interval and traffic flow flexibly according to the traffic conditions. The signal from the traffic light maintains order in the road using a control system to control the traffic flow (i.e., start or stop). Devices in the manufacturing line wait in an idle state until receiving the start signal from the PLC to perform a task. When the device finishes the task, the device sends the finish signal to the PLC and PLC continues the process using this signal.

The objective of this paper is to validate PLC code of the AS/RS using 3D simulation environment based on work-site data. Figure 3 illustrates a construction concept of a PLC simulation environment. The left side of the figure shows the virtual simulation environment and the right side shows the PLC code used in the real factory. The virtual environment consists of a logical model (I/O model) simulator (Park et al. 2008) and a graphic simulator modeled from PLC code symbol and actual survey data.

The 'PLC simulator' performs the simulation of the control program, and the 'plant model visualizer' shows the corresponding plant model (3D graphic models) reflecting the changing states of the production system during the PLC simulation. Thus, it becomes much easier for users to verify the PLC program though visualization the plant model.

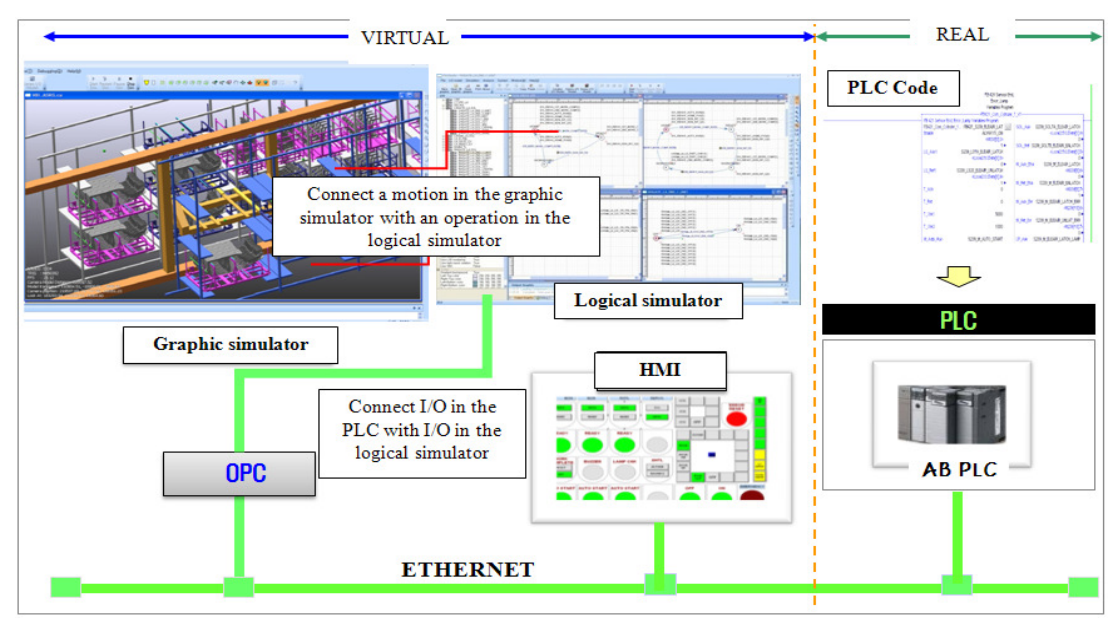

Figure 3: PLC simulation environments for AS/RS 
(1) Logical simulator (I/O simulator): A logical model is a kind of discrete event system model using input and output signals that is connected with PLC code. Therefore, users can make core logic used in the AS/RS with a logical model (Park et al. 2008).

(2) Graphic simulator: The 3D model based on the actual dimensions value in the real device is a resource for constructing a graphic simulator. It plays the role of an actual device in the virtual plant model.

(3) OPC: An acronym for 'OLE for Process Control', OPC plays a role of a connection between the PLC input, output points and the logical simulator.

(4) HMI: An acronym for 'Human Machine Interface', OPC is a user interface for the local control console to perform control using PC.

(5) AB PLC: The hardware PLC used in the factory made by Allen-Bradley receives the input signal and makes process of logic in the PLC code to export output signals.

(6) ETHERNET: A set of network cabling and network access protocol standards for a bus topology that plays a role of a pathway between PLC hardware and a virtual factory.

\section{CONSTRUCTION OF THE LOGICAL MODEL}

The plant model includes all manufacturing devices of the AS/RS as well as the corresponding device programs to perform their tasks in the production system, and the PLC program contains the control logic for the plant model. For integration of the plant model and the PLC program, it is necessary to define the mapping between the plant model and the PLC program, which is described by Park's logical model (I/O mapping model) (Park et al. 2008). The logical models used in the AS/RS mainly consist of three factors, the I/O signal of a stoker crane, a rack room position sensor, and a pallet state. The proposed simulation environment of the AS/RS is constructed using a number of logical models to define the mapping between the AS/RS plant model and the PLC program. The logical model is specified by a 9-tuple (Zeigler 1984, Kim 1994).

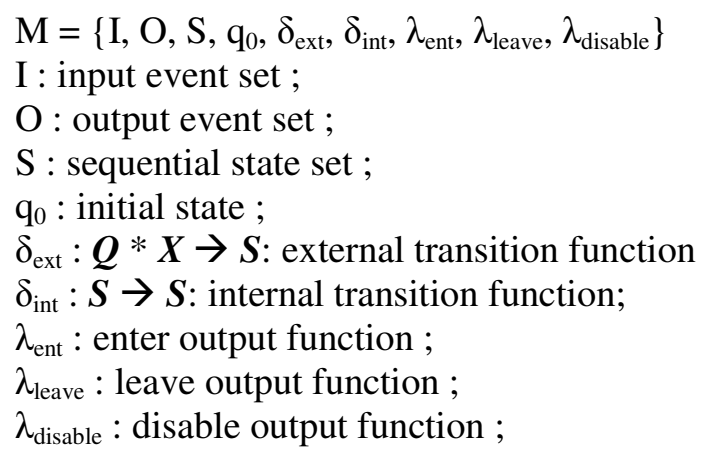

The results from constructing a logical model are divided into a set of input states and a set of output states. Functions $\left(\delta_{\text {ext }}, \delta_{\text {int }}, \lambda_{\text {ent }}, \lambda_{\text {leave }}, \lambda_{\text {disable }}\right)$ determine a state transition and interact with the PLC using an I/O signal. To describe AS/RS information based on the logical model, a model has to be constructed by an X-axis (the location of stoker-crane), a Y-axis (the location of rack room) and a Z-axis (situation of pallet). Figure 4 shows the results of the logical model. Each symbol and location information correspond with the real parameters used in the PLC.

The logical model is made by using the application tool "PLCStudio", a software package designed to validate the PLC program (UDMTek 2008, Park 2006). The PLCStudio presents a virtual environment 
for PLC code validation where the user can simulate the PLC code. The symbol descriptions used in figure 4 are as follows (see Table 2).

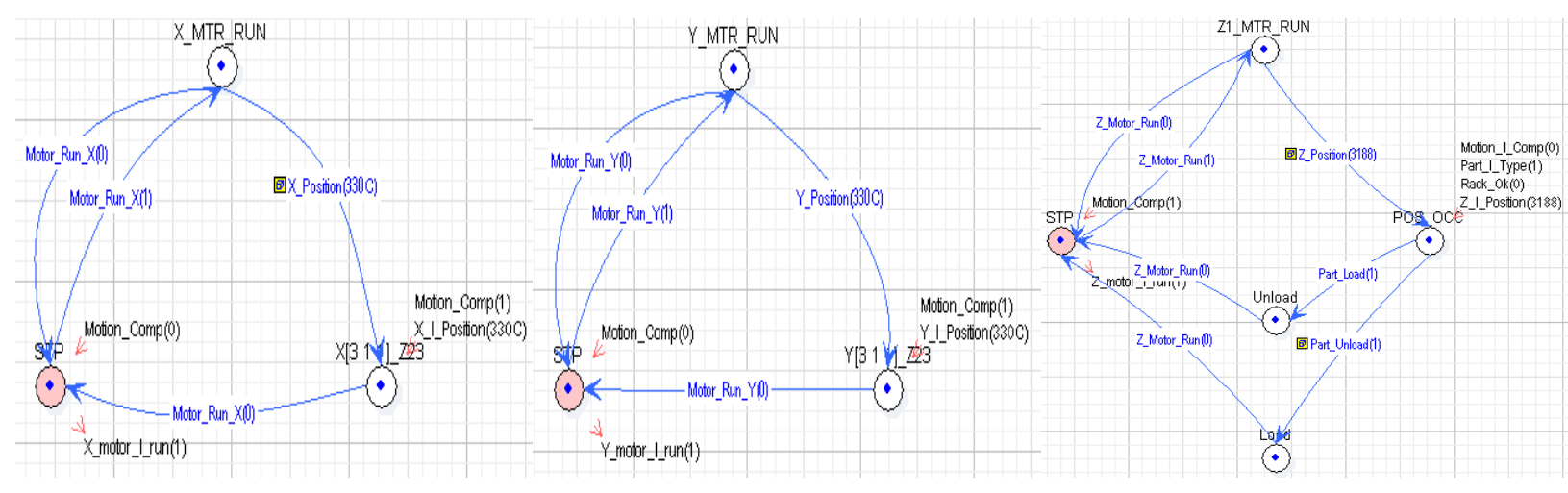

Figure 4: Logical models for AS/RS

Table 2: Description of implemented logical model symbol

\begin{tabular}{|c|c|}
\hline SYMBOL & DESCRIPTION \\
\hline *_Motor run & The working signal of a motor about each axis. \\
\hline *_Motor_I_run & A PLC input tag of *_Motor_run. \\
\hline *_Position & The location information of a axis. \\
\hline *_I_Position & A PLC input tag of *_Position. \\
\hline Part_I_Type & The panel type receiving from stoker-crane. \\
\hline Rack_Ok & $\begin{array}{c}\text { The signal expressing whether panel was loaded in the rack } \\
\text { or unloaded from the rack. }\end{array}$ \\
\hline
\end{tabular}

\section{CONSTRUCTION OF AN AS/RS 3D CELL}

This chapter describes how the AS/RS 3D cell can be constructed using the graphic simulator module of PLCStudio. The 3D cell is a kind of plant model (Hausma et al. 1976) that consists of manufacturing devices with their positions in the layout. To represent the manufacturing devices, this paper uses the concept of a virtual device, that is, a digital model imitating the physical and logical aspects of the AS/RS. A virtual AS/RS cell needs to maintain its relationship with the PLC code and should also retain attributes of the device, such as the kinematics and geometric shape.

The 3D model receives a starting signal from the logical model that executes a motion and then, sends an end signal to the logical model when the motion ends. That is, for validation of the control logic of the AS/RS, it is necessary to have a plant model enabling visual validation of the PLC program.

The implemented AS/RS 3D cell consists of two models: a non-kinematic model, a kinematic model. A 3D pallet model and a 3D panel model are non-kinematic models and the stoker-crane is a kinematic model (Figure 5). 


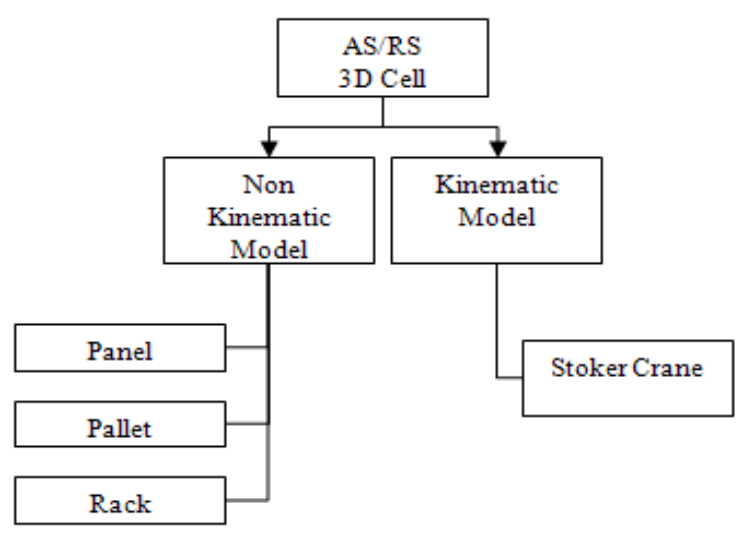

Figure 5: AS/RS 3D cell hierarchy

As shown in figure 6, there are three non-kinematic components in the 3D AS/RS cell: panels used in the automobile body assembly line, pallets to store the panels, and racks that store pallets. A panel has to be put to be placed on the correct pallet that has a specific shape in proportion to the panel and grooves to hold a certain number of panels. Thus, the color of the pairs and the panel pallet are determined according to the part of the car type for classification in an AS/RS cell.

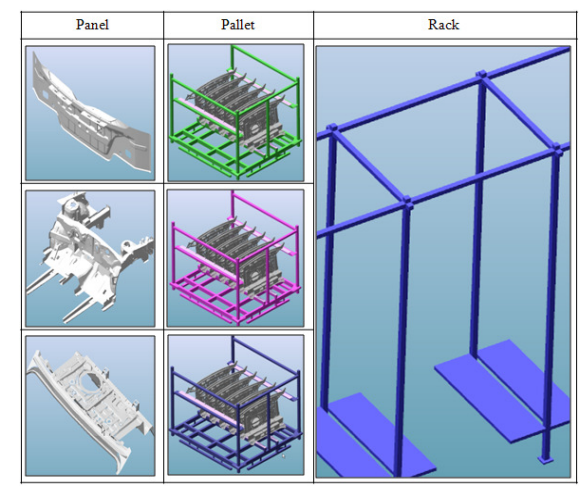

Figure 6: The non-kinematic 3D model

Figure 7 shows the 3D model features and kinematics information of the stoker-crane. The stokercrane can move according to a PLC input signal that indicates a specific X, Y, Z position address on the rack. The stoker-crane contrasts with the non-kinematic 3D model in that it requires a kinematic structure, including joints, axes, links, and velocity, to move according to the address signal. To move to a specific position on the rack, the stoker crane has to have moving factors, which consist of three kinds of joints, as shown in figure 7 . They are:

(1) X-Joint: X-Joint indicates the width coordinate in the cell and it has a moving range corresponding with the device joint limit range. The X-Joint signal from the PLC is a real number form that indicates the $\mathrm{X}$-coordinate of the center of a rack room.

(2) Y-Joint: Y-Joint indicates the height coordinate in the cell like the X-Joint and it points to a Ycoordinate of the bottom of each floor the pallet is located.

(3) Z-Joint: Z-Joint indicates the depth coordinate, and consists of four bars (Z1, Z2, Z3 and Z4). Four bars have two types of configuration depending on the pallet size because the pallet width can be divided into "Default pallet width" and "Side panel pallet width" as shown in Figure 8. 
The default pallet size means that the widths of all kinds of pallets, except for the side pallet, cannot exceed the default pallet width.

(a) (Z1-Z1: Z2-Z3): This configuration is used for carrying two default size pallets simultaneously. If the stoker-crane wants to alternate an empty pallet in the rack with a full pallet, a full pallet is located in (Z1-Z1) and an empty pallet in (Z2-Z3).

(b) (Z1-Z1-Z3): This configuration is used for carrying a side panel pallet. The side panel pallet is larger than other pallets because of the panel size. Thus, the if stoker-crane wants to alternate an empty pallet with a full pallet, the crane uses a "Z1-Z1-Z3" configuration to find the center of gravity of the pallet.

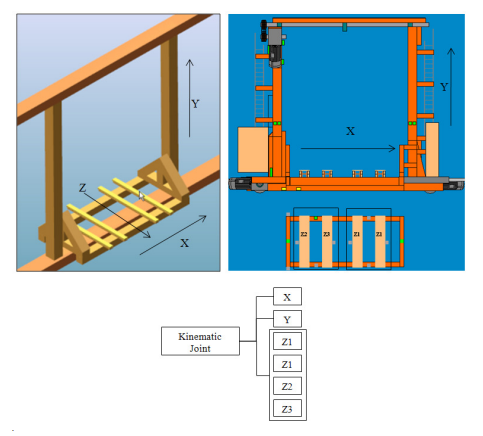

Figure 7: Kinematic 3D model

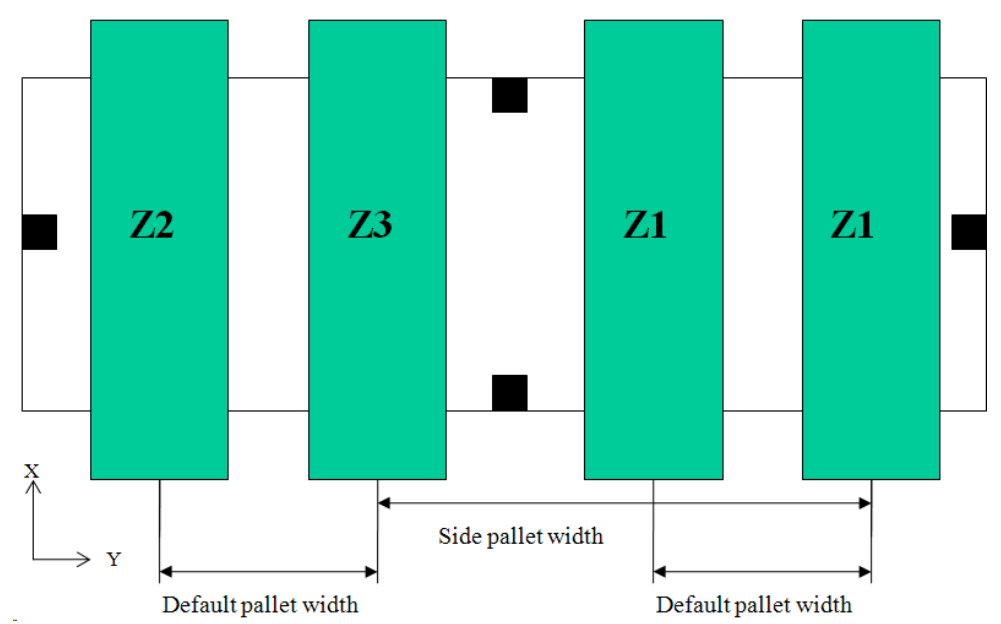

Figure 8: Pallet size and configurations used in AS/RS

The stoker-crane moves to the addressed rack location using an X, Y coordinate signal from the PLC and then selects or places a pallet using "Fork (Z-Joint)". Because this mechanism is based on the AS/RS operation used in the real factory, the graphical validation is the same as a test run of the AS/RS in the factory.

The 3D file used in the 3D AS/RS cell is converted from CATIA V5, Solid Edge. The 3D panel obtained from CATIA V5 and the 3D pallet obtained from Solid Edge is real data used for the car body and facility design. The rack and stoker-crane model are designed using PLCStudio based on a 2D drawings. All 3D model dimensions corresponds with real data used in the real factory. There are two major advantages of using real data in the 3D AS/RS cell: 1) there is no additional work to adjust the 3D model scale. 2) the motion distance of the stoker-crane is more realistic, because the rack address data received from the PLC indicates the rack room's coordinates. 
The aspects of the approach described in Sections 2 and 3 have been successfully implemented using an open-source code development platform, and the graphical outcome of a small-scale demonstration will be demonstrated at the conference site.

\subsection{Making 3D Simulation Events}

As noted above, the fork lift and the robot's activities are not addressed in this paper. Thus, these two constraints are related with events that occur artificially for the cell level simulation.

Figure 9 shows a pallet creation event and a pallet extinction event occurring in the cell. In the real work cell, the fork lift extract s the pallet from the rack when an alarm informs the empty pallet is located in the exit location. Because this paper does not consider the fork lift factor, this is treated as an event to construct a realistic simulation environment.

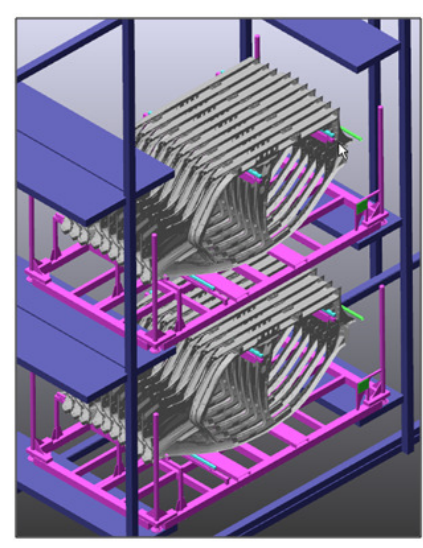

(a) Pallet creation event

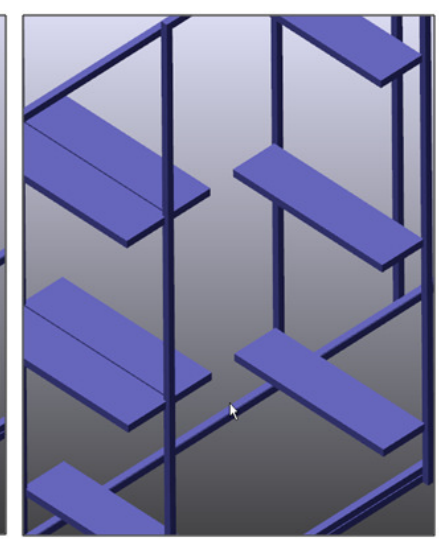

(b) Pallet extinction event

Figure 9: Simulation event creation

\section{CONCLUDING REMARKS}

This paper proposes an architecture for a PLC code validation environment for the AS/RS. The suggested approach enables visual verification of a PLC program by synchronizing the PLC program with a corresponding virtual plant model. The environment of the proposed method consists of three models: a virtual AS/RS model (plant model), a PLC program and a logical model (I/O mapping model). The virtual AS/RS model includes all manufacturing factors used in the AS/RS, and the PLC program contains the control logic for the virtual AS/RS model. The logical model functions as a communication link between these two models. Because the proposed architecture uses virtual devices and panel data and the PLC program used in a real factory, it is more realistic than other AS/RS simulation approaches using the Mehta model (Kang et al. 2006). The proposed methodology for the construction of a virtual AS/RS model presents an off-line PLC code validation environment. It fulfills the role of a test run in a real factory, and such it can increase the accuracy and simplify the logic before it is installed in the production environment. 
Ko, Shin, Wang, and Park

\section{ACKNOWLEDGMENTS}

This work was partially supported by the Defense Acquisition Program Administration and the Agency for Defense Development, Korea, under the Contract Number UD080042AD.

\section{REFERENCES}

Han, M., L.F. Mcginnis, J.S. Shieh, and J.A. White. 1987. On Sequencing Retrievals in an Automated Storage Retrieval System. IEEE Transactions 19 (1): 56-66.

Hausma, W. H., L.B. Schwarz, and S.C. Graues. 1976. Optimal Storage Assignment in Automatic Warehousing System. Management Science 22 (6): 629-638.

Kang, J. Y., H.C. Lee, and I.S. Um. 2006. A Study for Design Optimization of an Automated Distribution Center using the Simulation and Metamodel. Journal of the Korea society for simulation 15 (3) : 103114.

Kim, T. G. 1994. DEVSIM++ User's Manual. Department of Electrical Engineering, KAIST.

Lee, W. B. and C.H. Roh. 2006. Design of A PLC Program Simulator for nuclear Plant Using Compiler Technology. Journal of the Korea society for simulation 15 (1) : 11-17.

Pack, C. M. and K.Y. Seong, S.C. Park, and G.N. Wang. 2008. Simulation based Control Program Verification in an Automobile Industry. Proceedings of the 27th IASTED International Conference on Modelling, Identification and Control.

Pack, S. C., C. M. Pack, and G.N. Wang. 2008. A PLC programming environment based on a virtual plant. The International Journal of Advanced Manufacturing Technology 39 : 1262-1270.

Park, C. M. 2006. Development of Virtual Simulator for Visual Validation of PLC Program. International Conference on Computational Intelligence for Modeling Control and Automation. 32-36.

UDMTek. 2008. PLC Studio $\mathrm{R}$ product introduction <www • udmtek . com> .

Zeigler, B. P. 1984. Multifacetted modeling and discrete event simulation. Academic Press, Orland.

\section{AUTHOR BIOGRAPHIES}

MIN S. KO is a doctor's student in the Division of Industrial and Information Systems Engineering at Ajou University. His research interests include CAD/CAM, discrete event system modeling \& simulation, and digital manufacturing system. His email is <sebastianminsuk@gmail.com> .

HYE S. Shin is a master's student in the Division of Industrial and Information Systems Engineering at Ajou University. Her research interests include CAD/CAM, discrete event system modeling \& simulation, and digital manufacturing system. Her email is $<$ h. . shin83@gmail. com $>$.

G.N. WANG is an Professor in the Division of Industrial and Information Systems Engineering at Ajou University. His research interests include CAD/CAM, discrete event system modeling \& simulation, and digital manufacturing system. His email is <gnwang@ajou.ac.kr>.

SANG C. PARK is an Associate Professor in the Division of Industrial and Information Systems Engineering at Ajou University. Before joining Ajou, he worked at DaimlerChrysler Corporation and CubickTek Co., developing commercial and in-house CAD/CAM/CAPP/Simulation software systems. He received his BS, MS and Ph.D. degrees from KAIST in 1994, 1996 and 2000, respectively, all in Industrial Engineering. His research interests include CAD/CAM, discrete event system modeling \& simulation, and digital manufacturing system. His email is <scpark@a jou.ac.kr $\rangle$. 